\title{
Enabling Intelligence in Spectrum Sharing
}

This paper was downloaded from TechRxiv (https://www.techrxiv.org).

\section{LICENSE}

CC BY 4.0

SUBMISSION DATE / POSTED DATE

04-01-2022 / 07-01-2022

CITATION

Wu, Chi-Jen (2022): Enabling Intelligence in Spectrum Sharing. TechRxiv. Preprint. https://doi.org/10.36227/techrxiv.17811617.v1

$\mathrm{DOI}$ 


\title{
Enabling Intelligence in Spectrum Sharing
}

\author{
Chi-Jen $\mathrm{Wu}$ \\ Department of Computer Science and Information Engineering \\ Chang Gung University, Taiwan \\ email: cjwu@mail.cgu.edu.tw
}

\begin{abstract}
We argue that the capital expenditures made by an individual mobile network operator is extremely high and risky. Also, radio spectrum sharing still lacks intelligence in the current architecture of mobile networks and needs to be rethought. We propose that the goal for a disruptive innovation, in the future mobile network architecture, that shall be able to free mobile network operators from having to hold spectrum licenses and natively enable intelligent radio spectrum sharing among multiple mobile network operators. On the basis of the design principles, the duty of a single mobile network operator is split into two roles, one focuses on infrastructure development, the other only contains authorizations on the radio spectrum usage. We introduce a new role to the mobile network architecture, named Spectrum Trader, is a primary broker for spectrum trading, and it is used to coordinate with the demand-side requests and the supply-side resources to drive demand in a realtime bidding manner. We also introduce a spectrum embedding technique that shall enable efficient and intelligent spectrum allocation by recommending the right spectrum bands based on user scenario. Finally, several significant challenges that need to be addressed in practical deployment are investigated.
\end{abstract}

Index Terms - spectrum management, spectrum sharing, spectrum embedding, intelligent spectrum bidding

\section{INTRODUCTION}

With the exponential rise in mobile Internet traffic, the rapid proliferation of mobile devices creates a significant burden on today's mobile networks. Mobile Network Operators (MNOs) are facing with the challenges of devising new Radio Access Network (RAN) architectures, when the legacy RAN is becoming expensive and inadequate in satisfying the demands of future mobile trends [1]. Over the past decade, MNOs have had to deploy more and more base stations to deliver affordable and high quality mobile services, this has created a complex structure of ultra-dense heterogeneous base station networks. The mass deployment of base stations increases MNOs' capital expenditures in infrastructure because of the costs for business planning, hardware equipments and new site development, as well as operating expenditures due to the costs of maintenance and power usage.

Meanwhile, today's MNOs all suffer from the spectrum shortage issue, they have exclusively relied on specific licensed spectrum bands due to the limitations in today's mobile network architectures. For the most of MNOs, a solution to support the continued growth in mobile data traffic is to acquire new spectrum licenses to expand spectrum capacity. Morgan Stanley Research reported that the telecommunications industry spent about US $\$ 275$ billion on $4 \mathrm{G}$ and have yet to gain significant returns on the past investments. In fact, a major MNO has to raise more than several billions of dollars from spectrum bands to support mobile communication services. Unfortunately, mobile data traffic increase could be expected to outpace the spectrum resources of an individual MNO in the future 5G/B5G networks, and the spectrum scarcity situation will have occurred when the entire radio frequency spectrum is exhausted around 2035 [2].

Maximizing shared spectrum matters [3]. Spectrum is one of the most critical and biggest single assets for an individual $\mathrm{MNO}$, it is a critical resource for providing mobile communication services. A fundamental challenge is to efficiently exploit the underutilized bands of the static allocated licensed spectrum on the commercial deployment. Essentially, spectrum can be regarded as a national resource that is managed and owned by the government. The quantity of spectrum is a finite, if spectrum allocated in terms of frequencies. However, spectrum natively is a renewable resource that can be shared, the value of spectrum derives from how to share with other MNOs to maximize utilization.

An artificial intelligence ( $\mathrm{AI}$ )-enabled intelligent design of mobile networks architecture is needed to support the dynamic nature in spectrum management. Given the rise in system complexity and the exponential increase in the amount of data exchanged through mobile networks, we believe that it is time to rethink the design of current mobile network architectures fundamentally, and to drive modern AI technologies that act as enablers of such potential changes on solving the spectrum crunch and enabling intelligent spectrum sharing among MNOs.

We are inspired by the cutting-edge innovations of sharing-economy and deep learning models [4] in Natural Language Processing (NLP) research areas, and we are interested in how to design a sharing-economy innovation in telecommunications industry and the research issues of the proposed Intelligent Spectrum Bidding (ISB) architecture for real-time spectrum sharing in the future mobile Internet.

In the conceptual idea of ISB, instead of a single MNO (holding accountable for deploying infrastructures 
and maintaining spectrum access), the duty of a current MNO shall be split into two roles, one (MNO) focuses on infrastructure development, the other (Spectrum holder) only contains authorizations on the radio spectrum usage. We leverage the concept of Real-Time Bidding (RTB) [5] in digital advertising, and introduce a new role to mobile network architecture, named Spectrum Trader (SpectrExchange), to coordinate with the demand-side requests (MNOs) and the supply-side resources (Spectrum stakeholders) to drive demand and create niche markets. The SpectrExchange is a primary broker-dealer for spectrum trading in a real-time bidding manner. More specifically, when the amount of spectrum resources is unavailable to support the anticipated demand, a MNO can request and bid more spectrum resource supply via SpectrExchanges to achieve the spectrum provisioning without the boundary limitations between individual MNOs and the spectrum stakeholders, and can achieve a lower-cost infrastructure and get into a new market faster.

We introduce a spectrum embedding technique, named spectr2vec algorithm, to represent the spectrum and user scenario characteristics into spectrum vectors and turn the high-dimensional spectrum allocation problems into spectrum recommendation problems. With the spectrum recommendation mechanism, MNOs can bid the right spectrum bands based on user scenario, serve their customers better.

The proposed ISB presents a fundamental change from the current architecture of mobile networks. To the best of our knowledge, this paper is the first study to devise the disruptive innovation for sharing the radio spectrum resources and offers a different perspective that restructures the current mobile network architecture to become an intelligent spectrum sharing market for the future mobile Internet.

The rest of this paper is organized as follows. We illustrate a view of the modern mobile network architecture and the crucial works on spectrum sharing technology in Section II. The motivation to the proposed ISB mobile network architecture is described in section III and the ISB marketing is presented in section IV. A broader discussion and open questions on the design of ISB are presented in sections $\mathrm{V}$. Conclusion is drawn in section VI.

\section{Mobile Network Today}

The 5G mobile network is already emerging in the industry and academia fields. The major reason why $5 \mathrm{G}$ network is faster is the larger spectrum bands it uses, and it takes advantage of the celebrated Orthogonal Frequency Division Multiplexing (OFDM) encoding. Also, the air interfaces for $5 \mathrm{G}$ have more flexibility and lower latency than 4G/LTE. The coding and channel access technologies succeed and achieved great improvements in the past decades. And the core network technologies have moved from circuit-switching to the all-IP evolved packetswitching. However, the high-level architectures of mobile

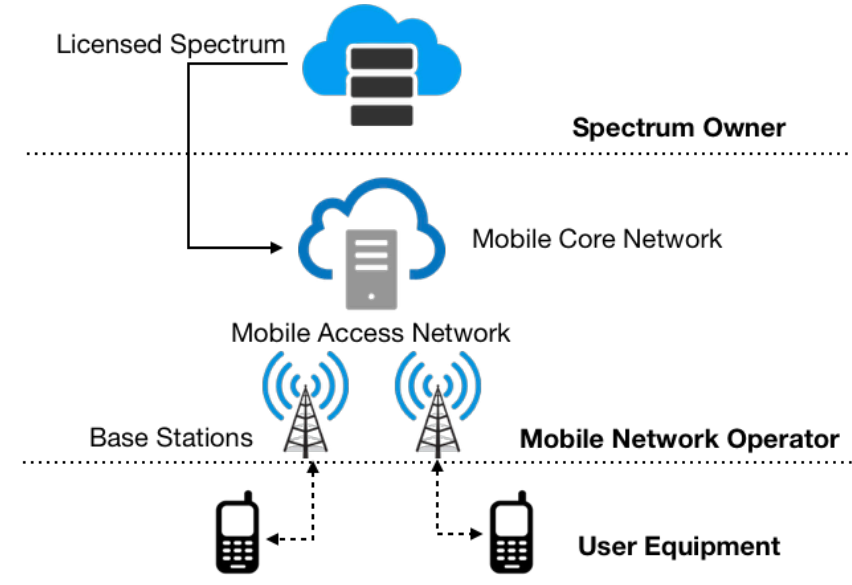

Fig. 1. A typical modern mobile network architecture

cellular network from $2 \mathrm{G}$ to $5 \mathrm{G}$ are no changes over the past 30 years. The Figure 1 illustrates a high-level architecture of a typical modern mobile cellular network. The modern mobile network architecture is based upon the interactions between three roles: spectrum owner, mobile network operator and User Equipment (UE). The interaction involves the three principal operations in the spectrum management: authorizing, equipping, and subscribing. The details of three roles are as follows.

Spectrum Owner: it is a spectrum manager, i.g., a governance regime, such as the Federal Communications Commission (FCC), manages the spectrum use in US. The trading and leasing are performed before the spectrum is accessed by a MNO. The most noticeable trading operation is the spectrum auction whereby a government uses an auction framework to sell the rights to transmit signals over the specific spectrum bands and to authorize the specified spectrum resources to a MNO.

Mobile Network Operator: it is a mobile communications company that equips and operates a mobile communication service for its subscribers. It needs to deploy a collection of complicated interdependent infrastructures, as roughly shown in Figure 1, including mobile core network, mobile access network and base stations. In addition, it controls access to a radio spectrum license from a government entity, and makes all equipped components available to its subscribers who want to use it.

User Equipment: a device used by a user has capability to communicate with base stations. A user has to subscribe and to be enrolled in the mobile communication service provided by a MNO. As shown in Figure 1, the UE mainly corresponds to the base stations in the mobile network architecture. The tough challenge is to carry a huge number of connected UEs. Mobility, the different usage scenarios and the heterogeneous UEs significantly increase the complexity of modern mobile network architecture. 


\section{A. Spectrum Sharing Technologies}

Spectrum sharing has been the research subject of intensive investigation within the past 20 years. The broader concept of spectrum sharing refers to the spectrum access technology that allows two or more mobile communication services using the same frequency bands. In the following we present an overview of key enabling technologies for spectrum sharing in the context of modern mobile communication networks.

In the current $5 \mathrm{G}$ deployment, dynamic spectrum sharing approaches [6] have become appealing solutions that allow the deployment of both $4 \mathrm{G}$ LTE and $5 \mathrm{G}$ New Radio (NR) in the same frequency band and dynamically allocates spectrum resources between the two technologies based on user demand.

To enhance spectrum sharing efficiency, database-driven technologies are appeared for already more than a decade age [7]. The concept of database-driven technologies is to access the band in a given location is controlled by one or more spectrum database systems, which are geographic databases that maintain information about the utilization and availability of the spectrum resources. The Dynamic Spectrum Access (DSA) [8] policy is one of the key strategies for mitigating the artificial spectrum shortage problem, and database-driven models have become the de facto solution for the realization of DSA. Several works of database-driven dynamic spectrum sharing model [9] have been shown to offer promise as a cost-effective and reliable approach for managing sharing among multiple classes of users with heterogeneous access rights and cognitive radio network technologies. In the view of DSA policy [8], a full featured DSA management should have technical, regulatory, business and market mechanisms in place to offer more flexible licensing management to enable spectrum resources to be dynamically reallocated and shared across services and MNOs.

Recently, a new architecture, Cloud Radio Access Network (C-RAN) [10] is proposed to meet such needs by centralizing the base stations and presenting a cooperative solution between multiple MNOs. The concept of C-RAN technology is similar to DSA with database-driven models, and comes with minimal cost, high energy efficiency, and a centralized cloud architecture that attracted a lot of attention both in academia and industry. Another example of sharing model is Licensed Shared Access (LSA) that allows additional spectrum access while protecting the incumbent user from harmful interference, and the implementations of LSA framework also rely on database-driven mechanisms. In the last 5G deployment, Citizens Broadband Radio Service (CBRS) is a shared-license band and deployed with a central database model. The databasedriven technologies can help AI technologies to identify the spectrum characteristics and to establish training models to sense spectrum status [11]. This article [12] surveys the recent progress in integrating AI technologies into Radio

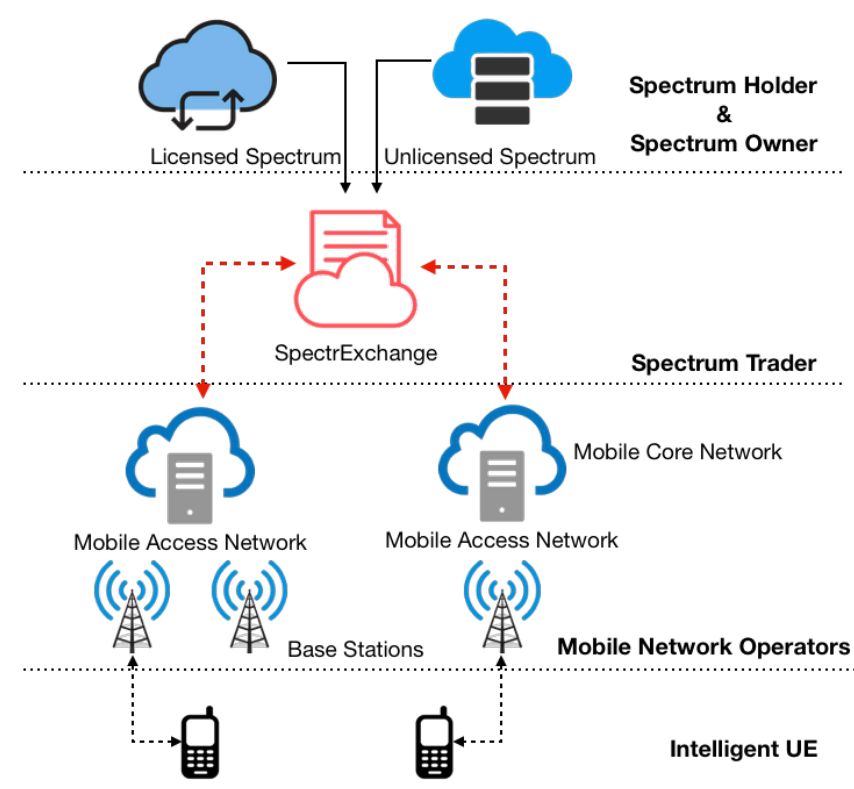

Fig. 2. The high-level architecture of ISB

Access Networks (RANs).

\section{An Intelligent Mobile Network}

Our ambition to convince the proposed ISB has the potential to fundamentally change the future mobile network architecture, which makes it possible to benefit from higher spectral efficiency offered by real-time spectrum bidding market. To achieve this vision, we promote fundamental research steps in modernizing legacy mobile network architecture and have set some assumptions regarding the interactions between main roles.

The Figure 2 illustrates the high-level architectural view of the proposed ISB mobile network. Here, we introduce the SpectrExchange to the new mobile network architecture. As mentioned earlier, we conceptually divide the duty of a current MNO into two separate abstract roles: MNO and spectrum holder. The MNO focuses on the mobile communication infrastructure development, and spectrum holder is accountable for authorizing the use of the radio spectrum. Besides the three basic roles, including spectrum owner, MNO and UE, the new architecture of ISB consists of five main roles which can offer the proposed real-time spectrum bidding market. We discuss the details of the five roles as follows (Spectrum owner and UE are the same as before).

SpectrExchange: it is a spectrum broker, as a stock exchange, which enables spectrum to be traded in an open way and is intended to coordinate with the bidding requests from the demand-sides (MNOs) and the spectrum resources from supply-sides (spectrum holders and owners) in ISB trading market. It is defined as a realtime marketplace that allows the buying and selling of the Spectrum Access Rights (SAR) for time slots (called SAR 
epoch or epoch for short) of the specific spectrum bands, and determines the specific SAR epoch to be allocated to the winning bidder (MNO). Both demand-sides and supply-sides are able to set criteria for the epoch they want to buy and sell. For all practical purposes, the design of SpectrExchange has to allow billions of epochs to be bought and sold in real-time, it is the most important building blocks for the ISB ecosystem success.

Spectrum Holder: it is an entity that holds exclusive licensed spectrum. The licensed spectrum refers to frequency bands used exclusively by MNOs for a set time period and expects to be protected from transmitting signals on the air within their licensed service areas. In ISB, spectrum holders, as well as spectrum owners (holds the unlicensed or shared-license spectrum), can partially transfer the usage of epochs on a voluntary commercial basis. Spectrum holder and owner both are spectrum stakeholders here.

Mobile Network Operator: it is a mobile communications company, but only concentrates on deploying mobile communication infrastructures. And now it is able to take advantage of ISB to provide a mobile service without holding the radio spectrum licenses. It sends a request to the SpectrExchange for temporary access to spectrum epochs as needed. A lack of entry barriers encourages a more competitive market in the provision of mobile communication services, it is analogous with the case of Wi-Fi. In the ISB architecture, a small MNO in a rural area will be possible to deploy its mobile communication service without handling the spectrum auction issue at early-stage investing. Thus, an entrant with a new business model has an incentive to develop its innovation without convincing the owner of the spectrum, and it returns the profits with the spectrum stakeholders via market mechanisms of ISB.

In the ISB architecture, the radio spectrum can be licensed or unlicensed. MNOs gain the rights to transmit by bidding permissions from the spectrum stakeholders as needed, and SpectrExchange serves as a delegated broker. The SpectrExchange and spectrum stakeholders can charge a fee for the shared spectrum, which provides spectrum stakeholders with an incentive to share more unutilized spectrum, and it builds up a sharing economy ecosystem with an incentive not to waste the spectrum.

Moreover, the MNO can get the quality of service guarantees in the exclusive epoch, a spectrum stakeholder shares the epoch if and only if it guarantees no interferences in this epoch. The ISB architecture makes guarantees available through explicit coordination between spectrum stakeholders and MNOs by SpectrExchange. It results in that MNOs have a motivation to engage with the SpectrExchange without fear that spectrum will be appropriated by others. Hence, MNOs can expect to make much profit with lower spectrum investment, and this will provide an incentive for MNOs to make complementary investments in building out a better network infrastruc-

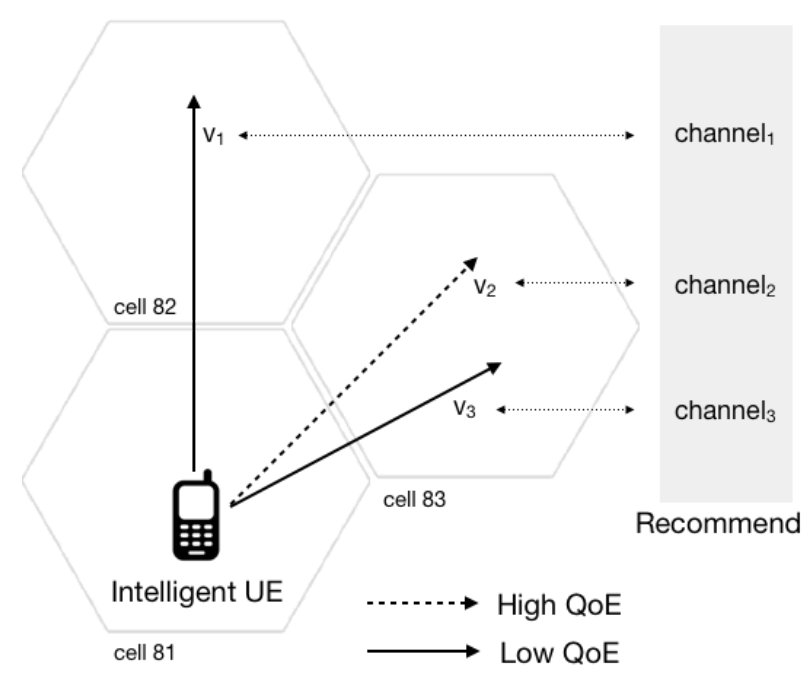

Fig. 3. An example of spectrum vector. For simplification purposes, there were two different trajectories, including $v_{1}$ and $\left(v_{2}, v_{3}\right)$ and two QoE requirements. Based on massive training data, the spectr2vec algorithm can achieve service recognition, traffic prediction and channel recommendation, e.g., channel $\mathrm{l}_{2}$ is recommended to $v_{2}$, since the spectrum characteristics of channel 2 are more suitable to the high QoE needs (high data rate, high reliability or low latency).

ture.

Next, we explain our two basic assumptions as follows.

Spectrum Access Right Epoch (SAR epoch): it is a basic trading unit between MNOs and spectrum stakeholders. We assume that a SAR epoch is the right to use spectrum in a period of time. We use a discrete interval of time model, and divide each channel of spectrum into small divisions (epochs) by frequency, geography (hex cells) and time. An individual request of bidding can be granted for a contiguous epoch. This way in which spectrum resources are abstracted can directly impact the spectral efficiency. Moreover, we also assume that the availability of an epoch is computed for each hexagonal cell in a predefined hex grid, as well as frequency and time dimensions. The initial pricing per epoch is also examined by the three dimensions of the epoch, e.g., an epoch is structured as a tuple (cell, channel, timestamp + offset, pricing) by appropriate actuarial models. And all MNOs should share the same map of the predefined hex grid.

Intelligent UE: smart phone is the great mass of traditional UE used to connect to the mobile Internet, but it will be replaced by a much broader variety of devices subsequently, and the user scenarios become various and diverse. We assume that in the near future, an intelligent UE can be produced, and it has a talent for representing the current description of user scenario with a particular list of numbers called a spectrum vector (spectrvec) for analyzing the characteristics of epoch utilization information and learning meaningful spectrum usage characteristics.

Therefore, we proposed the spectr2vec algorithm [13], a spectrum embedding technique uses a deep learning 
3

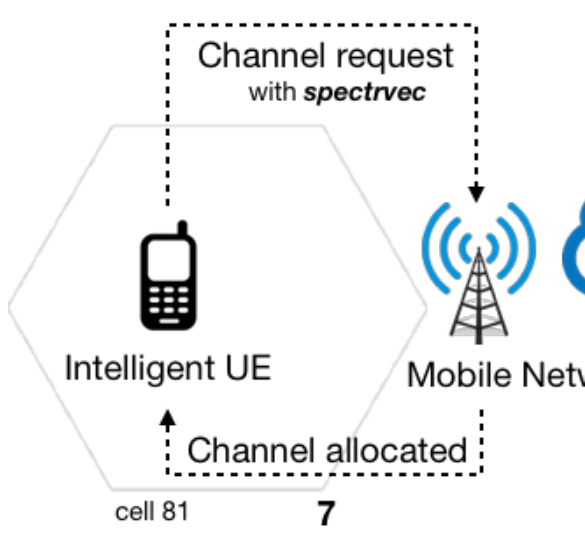

4

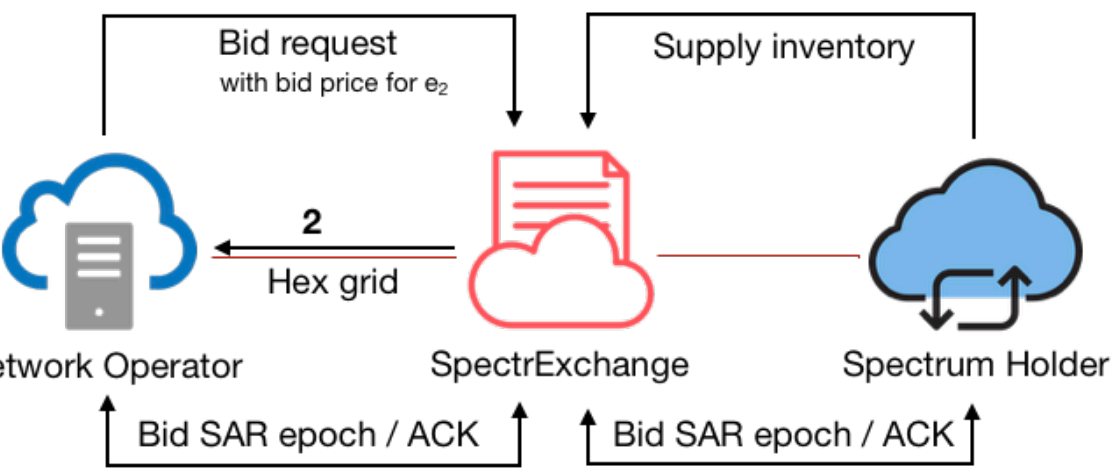

5

6

Time: 2021-06-25 19:10:00

Fig. 4. An auctioning example in the Intelligent Spectrum Bidding (ISB) ecosystem

model [4] to learn associations between spectrum allocation and QoE from a large set of scenario descriptors, including the GPS location, mobility, direction, altitude, velocity, priority, QoS requirements and the useful information for helping epoch recommendation. Once trained, such a deep learning model can utilize the historical knowledge to detect similar user scenarios or suggest epochs based on the application scenarios. We illustrate an example of the spectrvec concept in Figure 3. More specifically, we turn the spectrum allocation problem into a recommendation problem. The main reason is that the characteristics of the spectrum limit the range of applications, the design of the future mobile Internet should take into account.

\section{Intelligent Spectrum Bidding}

In a modern mobile network, the main challenge for sharing spectrum bands is how to determine which users can access the band and assign channels to them, all within a few milliseconds processing time. The proposed ISB leverages the concept of Real-time Bidding (RTB) [5] to achieve the requirements and holds for only a few milliseconds after a bid request is launched.

We illustrate a conceptual idea of ISB and describe the context by sketching the role each plays as the following scenario. We exploit a case of a user making a video call to describe the interactions between the major roles of ISB. For simplicity purposes, we assume only one SpectrExchange here. SpectrExchanges serve their clients spread across multiple geographic regions, such distributed clouds offer the possibility of hosting. We also assume that there is no available epoch offered from the spectrum owner in this instance. In Figure 4, the time is 2021-06-25 19:10:00. As a result, the inventory of epoch placement generated by the spectrum holder is like a list of tuples $t\left[e_{1}\left(\right.\right.$ cell $_{81}, f_{1}$, 2021-06-25 19:10:00+60s, \$0.1), e ecell $_{81}, f_{2}, 2021-06-25$ $19: 10: 00+600 \mathrm{~s}, \$ 0.8)$ ]. The user's location is in the range of the cell $_{81}$ in which two channels, $f_{1}$ and $f_{2}$, are available in the next $60 \mathrm{~s}$ and $600 \mathrm{~s}$, respectively. The initial pricing for the dedicated epoch is revealed in the inventory $(t)$.

To carry out a bidding process, three essential operations have to be initialized. First, the spectrum holder offers the inventory of epoch placement $(t)$ to the SpectrExchange, and has to register with the SpectrExchange as a stakeholder for this inventory $(t)$, that is shown as the step 1 in Figure 4 . Second, SpectrExchange incorporates all the received inventories into the predefined hex grid and marks all available epochs within the hex grid. Third, in the step 2 in Figure 4, SpectrExchange continuously syncs the hex grid to the associated MNOs.

When a user makes a video call, the intelligent UE is triggered to summarize the spectrvec instantly (or maintaining it periodically) by the spectr2vec algorithm [13]. The intelligent UE utilizes a control channel to communicate with the base station of the MNO and to send a message of demanding data channels, which contains the spectrvec to the base station (step 3). The MNO real-timely processes the all requests from subscribers, sums up the bid price for each request of bid, and then sends bid requests to the SpectrExchange (step 4). Here, the data channel, as opposed to a control channel that carries signaling information, will be released when the UE remains idle for a predetermined period of time. The descriptions of the step $\mathbf{3}$ and $\mathbf{4}$ in Figure 4 are as follows.

With massive spectrvec of channel requests, the MNO has the potential of compiling the spectrvec of bid requests to consolidate into an analysis for recommending which epoch will be involved in the auction. In the step 3 of 
Figure 4, the channel request is initiated by a video call (by revealing the QoS requirements in spectrvec), an AI-based pricing approach can be used to choose which epoch it takes based on the information, including the spectrvec and the info of epochs. Obviously, the $e_{2}$ is with a much better pricing option than $e_{1}$, and the time period of availability in $e_{2}$ is more appropriate for video calls. The bid price for $e_{2}$ can be determined through negotiation based on the current user density in cell $_{81}$ and the priority status of the subscriber; then the bid request is sent to SpectrExchange (step 4).

Honestly, many cut-edge hardware and software technologies are involved in a mobile network architecture. Due to limited space, a lot of details in base stations, radio access networks, and core networks are ignored here.

A competitive auction-based mechanism allows SpectrExchange to make real-time and data-driven decisions. Learning from analyzing massive bid requests, SpectrExchange has the capability of matching the best epoch and pricing the epochs through an intelligent auction-based scheme based on overall information exposure. It is noted that the MNOs may decide to offer different pricing for epoch auction, implying a priority ranking on the available epochs. Thus, SpectrExchange can gather all serious bids into an auction and pick the bid winner who has the best pricing for an epoch. The whole auctioning process in SpectrExchange should occur only in milliseconds.

After a bidding process closure, the SpectrExchange announces the bidding result (step $\mathbf{5}$ and $\mathbf{6}$ ), and the spectrum holder and the MNO are expected to acknowledge the bidding result to confirm the bid has been recorded within an instant period of time, as shown in Figure 4 .

The step 7 in Figure 4 is the last step in a bidding process. The MNO allocates the data channel listed in the epoch to the UE for making the video call. The base station of the MNO establishes the channel for the UE and enables the bearer service over the reserved data channel.

Someone may argue that a drawback of auctioning spectrum on the fly results in that such spectrum sharing system might have poor responsiveness and poor scalability. But it should be not at all certain that 1) MNOs can auction the epoch in advance before their subscribers require the spectrum bands based on usage patterns; 2) for the state-of-the-art RTB design, the Trade Desk company [14] shows that its RTB system can achieve 11 million queries per second and 800 billion queries per day.

Moreover, leaving bands of spectrum unlicensed leads to that spectrum owners (governments) might sacrifice the revenue obtained by auctioning spectrum licenses. By leveraging the ISB market, spectrum stakeholders can gain a market advantage and be encouraged to increase the supply of spectrum resources. In a nutshell, the ISB market can benefit for all involved parties. Spectrum stakeholders can increase revenues by offering epoch inventories for auctioning, including licensed and unlicensed spectrum. MNOs can increase their service capacity and boost spectrum effectiveness. The priority subscribers are willing to pay more for a better service experience.

\section{Discussion and Future Research Directions}

Mobile network, which has a 30-year history is composed of the latest and legacy technologies, and is interdisciplinary science. Given the current state of mobile network ecosystem, a clean slate approach is difficult or impossible. However, the impact of a new technology might be dependent on how much effort it takes to realize conceptual designs to be a running system. We recognize that the first priority is incremental deployment by coexisting with current mobile networks, we have a discussion first. Transparency and differentiated service will be discussed. Several crucial future research directions will be related as well.

(i) Incremental deployment: in the practical deployment of ISB, a MNO needs to install a spectrum demand service that's dedicated to fulfill subscribers' requests, and to deal with the bidding process and billing tasks. A spectrum supply service has to be deployed for spectrum stakeholders. All are new services, including SpectrExchange, such changes can be achieved with acceptable changes in the legacy mobile network. However, our result is preliminary, the additional research is needed to investigate in more details of these new services in protocol-level. Here, the intelligent UE is not of vital importance, but makes great improvements. A possible alternative approach is to treat with the spectrvec at mobile cloud [15].

(ii) Transparency: it is all about ensuring that subscribers are unaware of the spectrum auctioning procedure during data communication. In any case it is clear ISB affects the transparency of connectivity for UEs relying on the consistency of channel allocation, and for the MNO which auctions spectrum smoothly. By this design, ISB may impose limitations on connectivity. Hence, an epoch pool manager should be designed to conduct these epochs in a MNO internally, and should have strategies for accommodating more active UEs within limited spectrum. In our vision, the ISB service should be a hidden service for the subscribers.

(iii) Differentiated service: it is about how to boost the priority of critical UEs in any rescue or emergency situation after a disaster. Assume that most of the public utility services are available. In that situation, mobile communication is usually not available for all UEs, regardless of priority. Our ISB philosophy has the capacity to enable the critical UE to be granted access privileges to data channels for emergency situations by simply boosting their priority level. It can offer a truly differentiated service and have power to shape the data traffic in RANs.

As we all know of course, there is still a significant amount of research work to tackle challenges and future research directions in the fundamental phase of the ISB market. Such as, all new spectrum sharing technologies face the same question: how to deal with the existing 
spectrum stakeholders or how to encourage the existing players to move on to the next game. Therefore, more research is needed on how to take security and privacy issues in ISB, as well as legal issues. The development of ISB may require clarification of legal issues, especially in different countries. Several mentioned components in ISB are required for further investigation. Given these user scenario data (spectrvec), how to leverage the modern AI technologies to initialize the first pricing of epoch auction in MNOs. The time granularity of an epoch also needs to be examined appropriately. An epoch entrustment mechanism for spectrum stakeholders has to be discussed. Another issue is how can cloud native computing and mobile edge computing help the deployment of ISB. And the spectrum bidding mechanism in SpectrExchange should be considered in the future work.

\section{CONClusion}

Increasing commercial access to shared spectrum is the critical need that emphasized by the US FCC. We illustrate a sharing-economy design thinking refers to a network system approach to understanding how to take an intelligent spectrum bidding market into use in the future mobile network architecture. We then introduce a spectrum embedding technique, spectr2vec algorithm, to represent the spectrum and user scenario characteristics into spectrum vectors and turn the high-complexity spectrum allocation problems into spectrum recommendation problems. Without doubt, users will quickly embrace sharing-economy services, they can gain the economic benefits explicitly. By benefiting from sharing-economy thinking, the creation of the ISB market has brought about a change in how people buy and sell the communication resources in the future.

\section{ACKNOWLEDGMENT}

The work was supported in part by the Ministry of Science and Technology of Taiwan, under Contracts MOST110-2222-E-182-003-. The authors would like to thank the anonymous reviewers for their valuable comments and suggestions to improve the manuscript.

\section{REFERENCES}

[1] W. Lehr, Economics of Spectrum Sharing, Valuation, and Secondary Markets. John Wilev \& Sons. Lt.d. 2020. [Online]. Available: htt.ns://onlinelibrary.wiley.com/doi/abs/ 10.1002/9781119551539.ch18

[2] T. Cogalan and H. Haas, "Why would $5 \mathrm{~g}$ need optical wireless communications?" in 2017 IEEE 28th Annual International Symposium on Personal, Indoor, and Mobile Radio Communications (PIMRC), 2017, pp. 1-6.

[3] R. Berry, M. Honig, T. Nguyen, V. Subramanian, and R. Vohra, "The value of sharing intermittent spectrum," Management Science, vol. 66, no. 11, nn .5242-5264, 2020. [Online]. Available: https://doi.org/10.1287/mnsc.2019.3437

[4] T. Mikolov, I. Sutskever, K. Chen, G. Corrado, and J. Dean, "Distributed representations of words and phrases and their compositionality," in Proceedings of the 26th International Conference on Neural Information Processing Systems - Volume 2, ser. NIPS'13. Red Hook, NY, USA: Curran Associates Inc., 2013, p. 3111-3119.
[5] "Real-time bidding," https://en.wikipedia.org/wiki/ Real-time bidding, accessed: 2021-09-30.

[6] "Breakthrough 5G data call using dynamic spectrum sharing to accelerate nationwide $5 \mathrm{G}$ deplovments." https: //www.ericsson. com/en/news/2019/9/ericsson-spectrum-sharing, accessed: 2021-09-30.

[7] P. Bahl, R. Murty, T. Moscibroda, and R. Chandra, "Senseless: A database-driven white spaces network," IEEE Transactions on Mobile Computing, vol. 11, no. 02, pp. 189-203, feb 2012.

[8] M. M. Buddhikot, "Understanding dynamic spectrum access: Models,taxonomy and challenges," in 2007 2nd IEEE International Symposium on New Frontiers in Dynamic Spectrum Access Networks, 2007, pp. 649-663.

[9] M. Hoyhtya, A. Mammela, A. Chiumento, S. Pollin, M. Forsell, and D. Cabric, "Database-assisted spectrum prediction in $5 \mathrm{~g}$ networks and beyond: A review and future challenges," IEEE Circuits and Systems Magazine, vol. 19, no. 3, pp. 34-45, 2019.

[10] M. Peng, Y. Sun, X. Li, Z. Mao, and C. Wang, "Recent advances in cloud radio access networks: System architectures, key techniques, and open issues," IEEE Communications Surveys Tutorials, vol. 18, no. 3, pp. 2282-2308, 2016.

[11] H. Yang, A. Alphones, Z. Xiong, D. Niyato, J. Zhao, and K. Wu, "Artificial-intelligence-enabled intelligent $6 \mathrm{~g}$ networks," IEEE Network, vol. 34, no. 6, pp. 272-280, 2020.

[12] Y. Sun, M. Peng, Y. Ren, L. Chen, L. Yu, and S. Suo, "Harmonizing artificial intelligence with radio access networks: Advances, case study, and open issues," IEEE Network, vol. 35, no. 4, pp. 144-151, 2021.

[13] C.-J. Wu, "When artificial intelligence meets spectrum sharing," in submitted for publication, 2021, pp. 1-6.

[14] "The Trade Desk achieves hvperscale for real-time bidding," https://aerospike.com/customers/the-trade-desk/, accessed: 2021-09-30.

[15] I. A. Ridhawi, S. Otoum, M. Aloqaily, and A. Boukerche, "Generalizing ai: Challenges and opportunities for plug and play ai solutions," IEEE Network, vol. 35, no. 1, pp. 372-379, 2021.

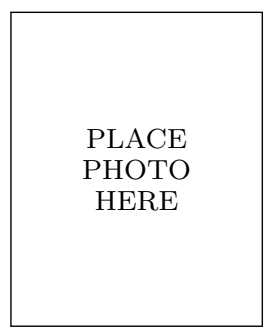

Chi-Jen Wu currently is an assistant professor of the Department of Computer Science and Information Engineering at Chang Gung University, Taiwan since February 2021. He received his Electrical Engineering Ph.D. from National Taiwan University in July 2012. He worked as a Distinguished Postdoctoral Scholar of the Institute of Information Science, Academia Sinica from 2012 to 2013. His research interests include Content Distribution, Mobile Cloud Computing, and Artificial Intelligence with a specific focus on Computational Advertising, Marketing Automation, and Financial Computing. 